\title{
BMJ Open Diagnostic and predictive accuracy of the Clinical Frailty Scale among hospitalised older medical patients: a systematic review and meta-analysis protocol
}

\author{
Aoife Leahy (D) , ${ }^{1,2}$ Margaret O'Connor, ${ }^{2}$ Jennifer Condon, ${ }^{3}$ Sarah Heywood, ${ }^{2}$ \\ Elaine Shanahan, ${ }^{2}$ Catherine Peters, ${ }^{2}$ Rose Galvin ${ }^{4}$
}

To cite: Leahy A, O'Connor M, Condon J, et al. Diagnostic and predictive accuracy of the Clinical Frailty Scale among hospitalised older medical patients: a systematic review and metaanalysis protocol. BMJ Open 2021;11:e040765. doi:10.1136/ bmjopen-2020-040765

- Prepublication history and additional materials for this paper is available online. To view these files, please visit the journal online (http://dx.doi. org/10.1136/bmjopen-2020040765).

Received 21 May 2020 Revised 26 November 2020 Accepted 09 December 2020

Check for updates

(c) Author(s) (or their employer(s)) 2021. Re-use permitted under CC BY-NC. No commercial re-use. See rights and permissions. Published by BMJ.

${ }^{1}$ School of Allied Health, University of Limerick, Limerick, Ireland

${ }^{2}$ Department of Ageing and Therapeutics, Univeristy Hospital Limerick, Limerick, Ireland

${ }^{3}$ Department of Medicine, University College Cork, Cork, Ireland

${ }^{4}$ School of Allied Health, Faculty of Education and Health Sciences, Ageing Research Centre, Health Research Institute, University of Limerick, Limerick, Ireland

Correspondence to

Dr Aoife Leahy;

aoife.leahy@ul.ie

\section{ABSTRACT}

Introduction Frailty is a common condition affecting older adults and is associated with increased mortality and adverse outcomes. Identification of older adults at risk of adverse outcomes is central to subsequent resource planning and targeted interventions. This systematic review and meta-analysis will examine the: (1) diagnostic accuracy of the Clinical Frailty Scale (CFS) in identifying hospitalised adults $\geq 65$ years with frailty and a medical diagnosis compared with the reference standard Frailty Index or Frailty Phenotype and (2) predictive value of the CFS in determining those at increased risk of subsequent adverse outcomes.

Methods and analysis We will include cross-sectional, retrospective and prospective cohort studies, and randomised controlled trials that assess either the diagnostic accuracy of the CFS when compared with the reference standard Frailty Index/Frailty Phenotype or the predictive validity of the CFS to predict subsequent adverse outcomes in hospitalised adults over 65 years with medical complaints. Adverse outcomes include falls, functional decline, unplanned Emergency Department attendance, emergency rehospitalisation, nursing home admission or death. A systematic search will be conducted in Embase, AMED, MEDLINE (Ebsco, Ovid, Pubmed), CINAHL, PsycINF0, Cochrane Library. Studies will be limited to those published from 2005 to 30 October 2019. Two independent reviewers will screen all titles and abstracts to identify relevant studies. The methodological quality of studies will be independently assessed using the Quality Assessment of Diagnostic Accuracy Studies-2. A CFS score of $>4$ will be used to identify frailty. We will construct $2 \times 2$ tables and determine true positives, true negatives, false positives and false negatives for each study when compared with the reference standard and for each adverse outcome. A bivariate random effects model will be applied to generate pooled summary estimates of sensitivity and specificity.

Ethics and dissemination Ethical approval is not required for this systematic review. We will disseminate our findings through a peer-reviewed journal.

\section{INTRODUCTION}

Frailty is a common condition that affects older adults across all settings of care. ${ }^{1}$ Clegg
Strengths and limitations of this study

- No previous reviews have been published exploring the predictive value of the Clinical Frailty Scale. Should a relevant review be published during the preparation of our review, it will be incorporated into our review and meta-analysis if relevant.

- Clinical and methodological heterogeneity across studies may limit our ability to draw reliable conclusions from the available evidence.

- We will employ standardised reporting guidelines to enhance transparency in conducting and reporting the systematic review.

et al describe frailty as 'a state of vulnerability to poor resolution of homeostasis following a stress and is a consequence of cumulative decline in multiple physiological systems over a lifespan'. ${ }^{2}$ It is a common presentation in Emergency Departments (EDs) but can be poorly defined due to ambiguity relating to its definition and pathophysiology. There are two current schools of thought relating to frailty. In the phenotypic model, frailty is viewed as a clinical syndrome with associated symptoms: unintentional weight loss, selfreported exhaustion, weakness, slow walking speed and low physical activity. ${ }^{3}$ Patients are usually defined as not frail, prefrail or frail. The second theory relates to the cumulative deficit model. Rockwood et al derived the Frailty Index whereby frail patients accumulate more conditions or symptoms associated with frailty. 'The more individuals have wrong with them, the more likely they are to be frail'.

Global demographics collated by the WHO indicate that more than $20 \%$ of the population will be aged over 60 years by $2050 .^{5}$ Recent findings from the Irish Longitudinal Study on Ageing (TILDA) demonstrated that $24.6 \%$ of patients over the age of 65 in Wave 


\begin{tabular}{lll}
\hline Table 1 & CFS scores \\
\hline & CFS 7 point scale & CFS 9 point scale \\
\hline Non frail & $1-4$ & $1-4$ \\
Frail & $5-7$ & $5-9$
\end{tabular}

CFS, Clinical Frailty Scale.

1 are living with frailty and $45 \%$ were prefrail as per the frailty index. ${ }^{1}$

There is an increasing body of literature demonstrating that frailty is associated with adverse outcomes including falls, functional decline, unplanned ED attendance, emergency hospitalisation, nursing home admission and death. ${ }^{34}$ Early identification of older adults at risk of such adverse outcomes through systematic screening can serve to identify high-risk groups in need of timely and targeted assessment and intervention either in the hospital or in the community setting. ${ }^{6}$ In 2011, Sternberg et al identified 20 different tools to identify frailty. ${ }^{7}$ Due to the heterogeneity of these tools, it can be difficult for the non-specialist to screen for frailty accurately and easily. Attempting to pick the appropriate tool is challenging due to a lack of consensus on a reference standard screening tool which is easily applicable to clinical practice. One of the more common screening tools used is the Clinical Frailty Scale (CFS) which was developed in Dalhousie by Rockwood et $a l$ in 2005. It was initially derived as a 7-point scale where individuals are classified as very fit to severely frail. It is based on functional status and underlying comorbidities. ${ }^{8}$ Due to the complex nature of severe frailty, the scale was extended to 9 points in $2008 .^{9}$

Table 1 illustrates the CFS. A score of $>4$ points indicates that the person is frail. Since its derivation, a number of studies have attempted to validate the CFS. The overall aim of this systematic review and meta-analysis is to examine the: (1) diagnostic accuracy of the CFS as a frailty screening tool compared with the reference standard Frailty Index or Frailty Phenotype in hospitalised adults $\geq 65$ years with medical complaints and (2) predictive value of the CFS in determining hospitalised adults $\geq 65$ years with medical complaints who are at increased risk of adverse outcomes.

\section{METHODS}

\section{Study design}

A systematic review and meta-analysis will be completed to identify relevant studies that assess either the diagnostic accuracy of the CFS when compared with the reference standard Frailty Index or Frailty Phenotype or the predictive validity of the CFS to predict adverse outcomes among hospitalised older adults with medical complaints. The review will adhere to the principles outlined in the Cochrane Handbook for Systematic reviews of Diagnostic Test Accuracy. ${ }^{10}$ We will also reference the Preferred Reporting Items for Systematic
Reviews and Meta-Analyses standardised reporting guidelines to standardise the conduct and reporting of the research. ${ }^{11}$

\section{Search methods}

A systematic search will be conducted in Embase, AMED, MEDLINE (Ebsco, Ovid, Pubmed), CINAHL, PsycINFO, Cochrane Library to identify relevant studies. A combination of the following keywords and search terms will be applied across the databases: Clinical Frailty Scale OR Rockwood Clinical Frailty Scale AND older adult OR retired OR geriatric OR aged OR aging OR elderly (online supplemental file 1) contains the precise search strategy for MEDLINE Pubmed. There will be no language restriction. We will limit our search to studies after 2005 when the CFS was first published. We will not search in grey literature or unpublished studies.

\section{Study selection}

Studies will be included based on the PEOSS (population, exposure, outcomes, study design and setting of care) criteria. The population of interest includes hospitalised older adults over 65 years of age. We will include studies with a predominantly older population (mean or median age of the population $\geq 65$ years). Our exposure of interest is the CFS. We will include studies that have examined the original 7-item CFS scale ${ }^{8}$ and the modified 9-item CFS scale. ${ }^{9}$ Outcomes of interest will include the diagnostic accuracy of the CFS when compared with the reference standard Frailty Index or Frailty Phenotype. In terms of the predictive accuracy of the CFS, adverse outcomes will include falls, injuries including fractures, functional decline, unplanned ED attendance, emergency hospitalisation, length of stay, nursing home admission and death. In the case of falls, we will be guided by the method and definition used to report falls in each individual paper. For example, we will take into account the rate of falls, number of falls and time to fall. We will include all cross-sectional (including baseline data from longitudinal studies), prospective and retrospective cohort study designs and randomised controlled trials. Setting of care will only include hospitalised medical patients. We will exclude abstracts which have not had a subsequent full-text peer-reviewed publication. We will also exclude studies the primary focus of which are patients in intensive care, surgical patients, patients with cardiac diseases, patients with renal disorders and patients with orthopaedic conditions.

All searches will be imported into endnote and duplicates will be removed. Two reviewers (AL and MO'C) will independently screen all titles and abstracts in the aforementioned databases for inclusion using the prespecified inclusion criteria. Where there are any disagreements regarding studies for inclusion, they will be resolved by a third independent reviewer (RG). A proposed data extraction table is included in the supplementary data (See online supplemental file 2). 


\section{Quality assessment}

Two reviewers (AL, MO'C) will independently review the methodological quality of each study using Quality Assessment of Diagnostic Accuracy Studies (QUADAS-2) tool. ${ }^{12}$ This tool consists of four key domains: Patient Selection, Index Test (CFS), Reference Standard (Frailty Index or Frailty Phenotype or adverse outcome) and Flow and Timing. For each individual study, the domains are evaluated in terms of risk of bias and the first three domains are evaluated with regards to concerns about applicability. Each of the independent reviewers will apply a rating of high, low or unclear across each of the domains through signalling questions included in the QUADAS-2 tool. If there is a conflict, it will be resolved by consensus where possible, in the event of no agreement, there will be a third independent reviewer (RG). The validity of each reported Frailty Index will be assessed using the criteria outlined by Searle et $a$. $^{13}$

\section{Statistical analysis}

We will conduct statistical analysis using Stata V.13. We will construct individual $2 \times 2$ tables and determine true positives, true negatives, false positives and false negatives for each study, for the reference standard and each adverse outcome. If additional data are required, we will contact the relevant authors to obtain the raw data in order to complete the $2 \times 2$ tables. We will calculate summary estimates of sensitivity and specificity with $95 \%$ CIs using the bivariate random effects model and plot them on a receiver-operating characteristic graph. Statistical heterogeneity will be explored using the variance of logit-transformed sensitivity and specificity, where smaller values indicate less heterogeneity between studies. In terms of the predictive accuracy of the CFS, Bayes' theorem will be used to estimate the post-test probability of an adverse outcome. ${ }^{14}$ The $c$ statistic or area under the curve, with $95 \%$ CI will be employed to describe model discrimination. Values between 0.7 and 0.9 represent moderate accuracy and values greater than 0.9 represent high accuracy. ${ }^{15}$ We will perform sensitivity analyses to examine the impact of methodological quality on the predictive value of the CFS. We will perform a subgroup analysis to explore the diagnostic accuracy of the CFS against each reference standard individually. Publication bias will be assessed using funnel plots.

\section{Certainty of the evidence}

We will use GRADE criteria to assess the certainty of the evidence from the meta-analysis. As per the GRADE guidelines, each study will be allocated a ranking of high or low based on the type of study. Each will then be upgraded or downgraded based on quality of the studies. ${ }^{16}$ The overall GRADE scores will inform our decision to make recommendations from our systematic review based on the quality of the studies included in the meta-analysis.

\section{Patient and public involvement (PPI)}

Patients and public were not involved in the conceptualisation or writing of the protocol for this review. The research questions addressed in this review arose from clinical observations of older adults. We will include PPI in the dissemination of our systematic review. We have established a PPI group of older adults at the Ageing Research Centre at UL and we will engage with a subgroup of this cohort of older adults who will review our paper and provide input.

\section{DISCUSSION}

The CFS is a common tool that is used to screen for frailty. This systematic review will investigate the diagnostic and predictive accuracy of the CFS for identifying frailty and adverse outcomes among hospitalised older adults with medical complaints. Our two reference standards, the Frailty Index and the Frailty Phenotype, reflect the deficit model and phenotypic model of measuring frailty. These two reference standards measure different constructs of frailty and we will examine the diagnostic accuracy of the CFS against both reference standards individually and collectively.

With an ageing population, increasing numbers of older patients are presenting to acute hospital services. Our proposed systematic review specifically explores the diagnostic and predictive accuracy of the CFS in an older hospitalised patient cohort with medical complaints The risk of subsequent adverse outcomes including functional decline and increased dependency has been reported to be high and often permanent among hospitalised older adults. ${ }^{17}$ Research indicates that frail older adults are more than twice as likely to experience a poor outcome than their non-frail counterparts. ${ }^{18}$ Identifying frail older adults who are at higher risk of adverse outcomes will support practitioners in targeting resource-intensive frailty interventions.

The reference standard approach to the management of frailty is the Comprehensive Geriatric Assessment (CGA). Ellis et al describe the CGA as a complex multifaceted approach where older adults are assessed and have appropriate intervention by specialist geriatric services in a holistic and multidisciplinary fashion. ${ }^{19}$ In a Cochrane review of CGA for older adults admitted to hospital, it was found that patients who had CGA were more likely to be alive and in their own homes at 3-12 month follow-up (RR: $1.06 ; 95 \%$ CI: 1.01 to 1.10$).^{20}$ Other interventions that have been explored with varying impact include physiotherapy, exercise regimes, nutritional support, multidisciplinary team input and pharmacological interventions. $^{21}$ This proposed systematic review will serve to explore the totality of evidence regarding the diagnostic and predictive accuracy of the CFS in identifying a highrisk hospitalised older medical cohort which may benefit from CGA.

Acknowledgements We acknowledge the contribution of the Patient and Public Involvement Group. 
Contributors $\mathrm{AL}, \mathrm{MO}$ ' $\mathrm{C}$ and $\mathrm{RG}$ were major contributors to drafting the manuscript. AL, MO'C and RG designed the study. AL developed the search strategy. SH, JC, ES and CP participated in the project design and critically appraised and edited the manuscript. RG is the guarantor of the review. All authors read and approved the final manuscript. The corresponding author attests that all listed authors meet authorship criteria and that no others meeting the criteria have been omitted.

Funding This project is funded by the Health Research Board of Ireland through the Investigator Led Project award 2017 (ILP-HSR-2017-014).

Competing interests None declared.

Patient consent for publication Not required.

Provenance and peer review Not commissioned; externally peer reviewed.

Supplemental material This content has been supplied by the author(s). It has not been vetted by BMJ Publishing Group Limited (BMJ) and may not have been peer-reviewed. Any opinions or recommendations discussed are solely those of the author(s) and are not endorsed by BMJ. BMJ disclaims all liability and responsibility arising from any reliance placed on the content. Where the content includes any translated material, BMJ does not warrant the accuracy and reliability of the translations (including but not limited to local regulations, clinical guidelines, terminology, drug names and drug dosages), and is not responsible for any error and/or omissions arising from translation and adaptation or otherwise.

Open access This is an open access article distributed in accordance with the Creative Commons Attribution Non Commercial (CC BY-NC 4.0) license, which permits others to distribute, remix, adapt, build upon this work non-commercially, and license their derivative works on different terms, provided the original work is properly cited, appropriate credit is given, any changes made indicated, and the use is non-commercial. See: http://creativecommons.org/licenses/by-nc/4.0/.

\section{ORCID iD}

Aoife Leahy http://orcid.org/0000-0003-2123-1513

\section{REFERENCES}

1 Roe L, Normand C, Wren M-A, et al. The impact of frailty on healthcare utilisation in Ireland: evidence from the Irish longitudinal study on ageing. BMC Geriatr 2017;17:203.

2 Clegg A, Young J, Iliffe S, et al. Frailty in elderly people. Lancet 2013;381:752-62.

3 Fried LP, Tangen CM, Walston J, et al. Frailty in older adults: evidence for a phenotype. J Gerontol A Biol Sci Med Sci 2001;56:M146-57.
4 Rockwood K, Mitnitski A. Frailty in relation to the accumulation of deficits. J Gerontol A Biol Sci Med Sci 2007;62:722-7.

5 Lutz W, Sanderson W, Scherbov S. The coming acceleration of global population ageing. Nature 2008:451:716-9.

6 Galvin R, Gilleit Y, Wallace E, et al. Adverse outcomes in older adults attending emergency departments: a systematic review and metaanalysis of the identification of seniors at risk (ISAR) screening tool. Age Ageing 2016;9:179-86.

7 Sternberg SA, Wershof Schwartz A, Karunananthan S, et al. The identification of frailty: a systematic literature review. J Am Geriatr Soc 2011;59:2129-38.

8 Rockwood K, Song X, MacKnight C, et al. A global clinical measure of fitness and frailty in elderly people. CMAJ 2005;173:489-95.

9 Clinical frailty scale, 2009. Available: https://www.dal.ca/sites/gmr/ our-tools/clinical-frailty-scale.htm

10 et alMacaskill P, Gatsonis C, Deeks J. Cochrane Handbook for systematic reviews of diagnostic test accuracy 2010. The Cochrane collaboration (online). Available: http:// dta.cochrane.org/handbookdta-reviews

11 Moher D, Liberati A, Tetzlaff J, et al. Preferred reporting items for systematic reviews and meta-analyses: the PRISMA statement. BMJ 2009;339:b2535.

12 Whiting PF, Rutjes AWS, Westwood ME, et al. QUADAS-2: a revised tool for the quality assessment of diagnostic accuracy studies. Ann Intern Med 2011;155:529-36.

13 Searle SD, Mitnitski A, Gahbauer EA, et al. A standard procedure for creating a frailty index. BMC Geriatr 2008;8:24.

14 Deeks JJ, Altman DG. Diagnostic tests 4: likelihood ratios. BMJ 2004:329:168-9.

15 Swets JA. Measuring the accuracy of diagnostic systems. Science 1988;240:1285-93

16 Goldet G, Howick J. Understanding grade: an introduction. J Evid Based Med 2013;6:50-4.

17 Heim N, van Fenema EM, Weverling-Rijnsburger AWE, et al. Optimal screening for increased risk for adverse outcomes in hospitalised older adults. Age Ageing 2015;44:239-44.

18 Dent E, Chapman I, Howell S, et al. Frailty and functional decline indices predict poor outcomes in hospitalised older people. Age Ageing 2014;43:477-84.

19 Ellis G, Langhorne P. Comprehensive geriatric assessment for older hospital patients. Br Med Bull 2004;71:45-59.

20 Ellis G, Whitehead MA, Robinson D, et al. Comprehensive geriatric assessment for older adults admitted to hospital: meta-analysis of randomised controlled trials. BMJ 2011;343:d6553.

21 Apóstolo J, Cooke R, Bobrowicz-Campos E, et al. Effectiveness of interventions to prevent pre-frailty and frailty progression in older adults: a systematic review. JBI Database System Rev Implement Rep 2018;16:140-232. 